\title{
Evaluating multipulse integration as a neural-health correlate in human cochlear-implant users: Relationship to spatial selectivity
}

\author{
Ning Zhou ${ }^{\text {a) }}$ \\ Department of Communication Sciences and Disorders, East Carolina University, Greenville, \\ North Carolina 27834, USA \\ Bryan E. Pfingst \\ Department of Otolaryngology, Kresge Hearing Research Institute, University of Michigan, \\ Ann Arbor, Michigan 48109-5616, USA
}

(Received 27 October 2015; revised 11 August 2016; accepted 19 August 2016; published online 7 September 2016)

\begin{abstract}
The decrease of psychophysical detection thresholds as a function of pulse rate for a fixed-duration electrical pulse train is referred to as multipulse integration (MPI). The MPI slopes correlate with anatomical and physiological indices of cochlear health in guinea pigs with cochlear implants. The aim of the current study was to assess whether the MPI slopes were related to the spatial spread of activation by electrical stimulation. The hypothesis was that MPI is dependent on the total number of excitable neurons at the stimulation site, with broader neural excitation producing a steeper threshold decrease as a function of stimulation rate. MPI functions were measured at all stimulation sites in 22-site electrode arrays in human subjects. Some sites with steep MPI functions and other sites with shallow functions were assessed for spatial spread of excitation at 900 pps using a forward-masking paradigm. The results showed a correlation between the slopes of the forwardmasking functions and the steepness of MPI, with broader stimulation predicting greater integration. The results are consistent with the idea that integration of multiple pulses in a pulse train relies on the number of excitable neurons at the stimulation site. (C) 2016 Acoustical Society of America.
\end{abstract}

[http://dx.doi.org/10.1121/1.4962230]

[ICB]

Pages: $1537-1547$

\section{INTRODUCTION}

In pulsatile electrical stimulation with cochlear-implant (CI) auditory prostheses, the temporal envelopes of the speech signal in each of the multiple adjacent frequency bands are coded by amplitude modulation of electrical pulse trains. Detection of an electrical pulse train is dependent on stimulation parameters such as pulse rate, phase duration, duration of the stimulus, and electrode configuration. Multipulse integration (MPI) in this context is defined as the decrease of detection threshold for a fixed-duration pulse train with the increase of stimulation rate.

For a fixed pulse-train duration, the number of pulses increases as the stimulation rate increases. The integration of multiple pulses in the pulse train, i.e., MPI, is quantified as the slope of the threshold-versus-pulse-rate function. In both guinea pig and human subjects with CIs, the MPI slope has been observed to be shallower for pulse rates below 300 pulses per second (pps) than for higher pulse rates, and the magnitude of MPI in this low-rate range was comparable to that of temporal integration (Zhou et al., 2015). These findings are consistent with the central temporal integration window having a size of approximately $3 \mathrm{~ms}$ corresponding to 300 pps (Viemeister and Wakefield, 1991; McKay et al.,

\footnotetext{
a)Electronic mail: zhoun@ecu.edu
}

2013). MPI slopes became steeper for rates above $300 \mathrm{pps}$ and steeper again as the pulse rate exceeded $1000 \mathrm{pps}$, where the residual cell-membrane partial-depolarization by subthreshold pulses is believed to facilitate neuronal activation at a lower current level in response to the subsequent pulses (Middlebrooks, 2004; Pfingst et al., 2011). Recent findings using alternating polarity pulses however suggest alternative mechanisms for rates above 1000 pps (Carlyon et al., 2015).

Several guinea pig studies have suggested that the ability to integrate multiple pulses is dependent on cochlearhealth variables such as the density of spiral ganglion cells (SGNs) near the stimulation site, residual hair cells in the corresponding frequency region, and the ensemble spontaneous activity of the participating neurons (Kang et al., 2010; Pfingst et al., 2011; Zhou et al., 2015). The MPI slope below $1000 \mathrm{pps}$ as a whole and its very low (5-156 pps) and middle rate (313-625 pps) segments each strongly correlates with the cochlear health variables, with steeper slopes predicting higher residual hair cell counts, higher SGN densities, and higher ensemble spontaneous-activity levels (Zhou et al., 2015). However, the correlation between MPI slopes in the extremely low-rate range ( $\leq 156 \mathrm{pps})$ and SGN density might be related to loudness growth but not neural density per se, because in this rate range, neural activity is unlikely to be limited by refractoriness (McKay et al., 2003). In cases of sparse neural survival, the current level required for reaching thresholds for the low-rate stimuli would be high, which 
could possibly result in an activation of the more densely packed neurons in the internal auditory meatus or auditory fibers with steep input-output (I/O) functions. A rapid increase in neural excitation as a function of stimulus level in these cases would predict shallow MPI slopes (McKay et al., 2003). That is, to maintain threshold (i.e., a certain amount of neural activity), the current level reduction needed to compensate for an increase in stimulation rate would be small, resulting in shallow MPI slopes. The fact that the absolute detection thresholds at very low rates ( $\leq 156 \mathrm{pps}$ ) correlate with MPI slopes in guinea pig data are consistent with this prediction (Zhou et al., 2015).

For higher rates $(>156 \mathrm{pps})$, individual auditory nerve fiber discharge rates are unlikely to follow the stimulation rate because they can be temporally limited by their refractory properties or accommodation to sub-threshold pulses, both leading to reduced neural excitability (Boulet et al., 2016). If the auditory fibers are not spontaneously active due to the absence of hair cells, at supra-threshold levels the fibers would all tend to fire at the first pulse in the pulse train and go into refractoriness in a synchronized fashion, resulting in an alternating high-low neural firing pattern to successive pulses in a high-rate stimulus (Wilson et al., 1997; Hu et al., 2003; Hughes et al., 2012, 2014; Ramekers et al., 2015). The steepness of MPI slopes, i.e., the rate of threshold decrease, depends on the increase of neural activity in the temporal integration window with the increasing pulse rate at a fixed stimulus current level. High neural density might offset the temporal constrains on the excitability of the single fibers by activating more neurons that are not in a refractory state, thus producing more neural activity as stimulation rate increases. At a stimulation site with low SGN density, there may not be enough neurons to be further recruited as rate increases, consistent with the association of low SGN density and shallow MPI slopes in this rate range (Kang et al., 2010; Pfingst et al., 2011; Zhou et al., 2015). For rates above $1000 \mathrm{pps}$, partial depolarization on the single-neuron level is thought to play a facilitative role in decreasing thresholds (Middlebrooks, 2004). MPI slopes in this rate range are only weakly correlated with the cochlear-health variables (Pfingst et al., 2011). We speculate that charge interaction and potentially other factors not necessarily affected by pathology (Carlyon et al., 2015), might mask the relationship between MPI slopes and neural survival at rates above 1000 pps.

In humans with CIs who do not have measurable residual hearing, MPI slopes have been shown to vary with similar magnitudes as those found in animals with a range of haircell conditions, suggesting that hair cells are not essential for MPI and that SGN density is more likely the anatomic variable that is responsible for MPI slopes (Zhou et al., 2012; Zhou and Pfingst, 2014). An important difference between previously published studies in guinea pig and human subjects with CIs is that the position of the electrodes with respect to the modiolus was relatively constant in the guinea pig experiments but varied considerably in the human subjects. In the animal studies cited above, the apical end of the electrode array, where the primary test electrode was located, typically filled much of the scala tympani space, allowing little variation in the distance of electrodes from the modiolus.
In such situation the number of excitable neurons should be strongly related to the local SGN density. However, if the position of the electrodes with respect to the modiolus varies from one electrode to the next, as it does in most human implants, the number of excitable neurons could vary as a function of both SGN density and the distance of the electrodes from the neurons. If MPI is dependent on the number of neurons activated, we hypothesize that steeper MPI slopes would, in general, be expected at sites with broader neural activation, assuming that there is reasonably consistent neural density in the stimulated region. Information about the relationship between spread of activation and MPI slopes is needed to help define the variables contributing to the variation in MPI slopes within and across human CI users.

\section{METHODS}

\section{A. Subjects and hardware}

Eight post-lingually deafened adult subjects participated in the study. Six of the eight subjects were sequentially implanted bilaterally. One of the six bilateral subjects (S99) was tested in both ears. The tested ears were implanted with the Nucleus ${ }^{\circledR}$ CI24RE or CI512 devices (Cochlear Corporation, Englewood, CO) and all were programmed with an ACE speech-processing strategy using the 900 pps default rate setting. None of the subjects had measurable residual hearing in the tested ear or in the non-implanted ear to the limits of the audiometer $(150 \mathrm{~Hz}: 70 \mathrm{~dB} ; 250 \mathrm{~Hz}$ : $90 \mathrm{~dB} ; 500 \mathrm{~Hz}: 100 \mathrm{~dB} ; 1000 \mathrm{~Hz}: 100 \mathrm{~dB} ; 2000 \mathrm{~Hz}: 100 \mathrm{~dB}$; $4000 \mathrm{~Hz}: 100 \mathrm{~dB}$; and $8000 \mathrm{~Hz}: 80 \mathrm{~dB}$ ). The demographic data for the nine tested ears are shown in Table I. The use of human subjects for this study was reviewed and approved by the University of Michigan Medical School Institutional Review Board and the East Carolina University Institutional Review Board.

\section{B. Psychophysical testing}

Laboratory-owned Nucleus ${ }^{\circledR}$ Freedom processors (Cochlear Corporation, Englewood, CO) were used for the psychophysical tests. The stimuli were delivered through MATLAB programs that communicated with the NIC II research interface. All stimuli were trains of symmetric biphasic pulses with a phase duration of $25 \mu \mathrm{s}$ and an interphase interval of $8 \mu$ s. Stimuli were presented in a monopolar (MP 1+2) electrode configuration.

\section{Measuring MPI slopes}

For each tested ear, psychophysical detection thresholds were measured for 2 pulse rates ( 80 and $640 \mathrm{pps}$ ) at a fixed pulse-train duration of $250 \mathrm{~ms}$. All available sites in the subjects' electrodes arrays were tested; sites turned off in the clinical processor map were not tested. Subjects were instructed to use the method of adjustment to find the lowest level at which the stimulus was detectable. Three sets of buttons in a graphic user interface were available on the computer screen, allowing up and down changes in 25, 5, and 1 clinical level unit (CLU). Subjects were instructed to raise the level of the stimulus to a level that produced loudness 
TABLE I. Subject demographics.

\begin{tabular}{|c|c|c|c|c|c|c|c|}
\hline Subject & Ear & Gender & Age (yrs) & CI use (yrs) & Duration of deafness $^{\mathrm{a}}$ (yrs) & Implant type & Etiology \\
\hline S60 & $\mathrm{R}$ & M & 71.4 & 2.2 & 6.2 & CI24RE(CA) & Progressive \\
\hline S81 & $\mathrm{L}$ & $\mathrm{F}$ & 59.8 & 5.2 & 2.5 & $\mathrm{CI} 24 \mathrm{RE}(\mathrm{CA})$ & Progressive \\
\hline S89 & $\mathrm{L}$ & M & 66.4 & 2.3 & 64.1 & CI24RE(CA) & Hereditary \\
\hline S69 & $\mathrm{R}$ & M & 70.8 & 1.6 & 9.2 & CI512 & Noise exposure \\
\hline $\mathrm{S} 88$ & $\mathrm{~L}$ & M & 60.0 & 2.2 & 4.5 & CI24RE(CA) & Progressive \\
\hline S93 & $\mathrm{L}$ & $\mathrm{F}$ & 64.0 & 6.0 & 26.0 & CI24RE(CA) & Progressive \\
\hline S94 & $\mathrm{R}$ & $\mathrm{F}$ & 71.5 & 7.8 & 11.5 & CI24RE(CA) & Unknown \\
\hline S99 & $\mathrm{L}$ & $\mathrm{F}$ & 48.0 & 1.6 & 41.5 & CI24RE(CA) & Hereditary \\
\hline S99 & $\mathrm{R}$ & $\mathrm{F}$ & 48.0 & 1.5 & 41.5 & CI24RE(CA) & Hereditary \\
\hline
\end{tabular}

${ }^{\mathrm{a}}$ Estimated duration of deafness prior to implantation.

that the subject could confidently detect and then decrease it close to a barely detectable level. This was done to avoid confusion between possible tinnitus percepts and the actual stimulus. For each pulse rate, two thresholds were estimated and the average was taken. The averaged thresholds from two repetitions were first converted from CLU to $\mathrm{dB}$ re $1 \mathrm{~mA}$ and MPI slopes were quantified in $\mathrm{dB}$ per doubling of pulse rate. Of all the MPI slopes measured, the two stimulation sites with relatively steep and shallow MPIs were further assessed for spatial selectivity of neural excitation. For three subjects (S60R, S89R, and S69L), two sets of steep and shallow MPI sites (four sites total) were chosen. The MPI slopes obtained from the two-rate, method-of-adjustment protocol used in this experiment were similar to those derived using more rates and adaptive procedures on the same electrodes in a study reported previously (Zhou et al., 2015).

For the chosen stimulation sites, we also estimated the distance between the electrode and the modiolus based on the average electrode-modiolus distance as a function of electrode number from Long and colleagues (2014, 2015). The electrode array used by the subjects receiving computed tomography (CT) scans by Long and colleagues (2014, 2015) was the same type as that used by our subjects.

\section{Measuring spread of neural excitation}

The chosen stimulation sites were evaluated for spatial selectivity of neural excitation using a forward-masking paradigm. If the masker and probe electrodes stimulate overlapping neural populations, the masker that is presented forward in time will cause some neurons in the population normally stimulated by the probe to enter a refractory period, thus elevating the threshold for detecting the probe stimulus. Forward masking generally diminishes as the spatial distance between the masker and probe increases (e.g., Azadpour et al., 2013). The masker used in the experiment was $300 \mathrm{~ms}$ long, followed by a $10 \mathrm{~ms}$ gap, and a $20 \mathrm{~ms}$ probe. A pulse rate of $900 \mathrm{pps}$ was used. The position of the probe was fixed while the position of the masker was varied from $2.25 \mathrm{~mm}$ apical to the probe to $2.25 \mathrm{~mm}$ basal to the probe, in steps of $0.75 \mathrm{~mm}$ (electrodes were spaced at $0.75 \mathrm{~mm}$ intervals). This included a condition in which the masker and probe were delivered to the same electrode $(0 \mathrm{~mm}$ interval). The level of each of the maskers was determined by loudness-balancing the masker to $50 \%$ of the dynamic range (DR) of the probe.
Threshold of the probe was measured first without a masker using a 3-alternative forced choice (3AFC) procedure, and with a randomly positioned masker within the designated tonotopic locations using a $3 \mathrm{AFC}$ procedure. The subjects were instructed to identify the one interval that sounded different than the other two, i.e., sound with a chirp in the end. The masker level remained constant, while the probe level started at $50 \%$ DR and adapted using a 2-down 1-up criterion. The step size was $10 \mathrm{CLU}$ for the first reversal, $5 \mathrm{CLU}$ for the second, $2 \mathrm{CLU}$ for the third, and $1 \mathrm{CLU}$ for the rest of the 9 reversals. The probe levels at the last six reversals were averaged and taken as the threshold. Forward masking was quantified as the amount of threshold elevation of the probe (in $\mathrm{dB}$ ) in the presence of a masker and plotted as a function of probe-masker distance. The forward-masking function was then divided by the threshold elevation at $0 \mathrm{~mm}$ (peak masking) to remove the factor of forward-masking recovery at $10 \mathrm{~ms}$ at the probe site such that the slope of the forward-masking function reflected spatial selectivity of neural excitation alone (McKay, 2012). The forward-masking slopes were calculated for the basal and apical sides of the function and were averaged. In cases where the probe was at the very end of the electrode array, such that the maskers were only placed in one direction to the probe, one forwardmasking slope was calculated.

\section{RESULTS}

Psychophysical detection thresholds measured for the two stimulation rates are shown in Fig. 1. Slopes of the MPI functions are shown in Fig. 2. MPI varied considerably across the electrode array for most ears tested. Integration was typically greatest in the middle of the array. Two stimulation sites indicated by circles were further assessed for spatial selectivity of neural excitation: one with relatively steep MPI and one with shallow MPI. Notice that for S60R, S89R, and S69L, an additional set of stimulation sites with the steep and shallow MPI were tested for forward-masking (indicated by squares).

The raw forward-masking functions with masked and non-masked probe thresholds are shown in Fig. 3. The normalized forward-masking functions (where each data point was divided by the magnitude of forward-masking at $0 \mathrm{~mm}$ ) are shown in Fig. 4. The masking functions, particularly those with steep slopes, were not always linear and in a few 

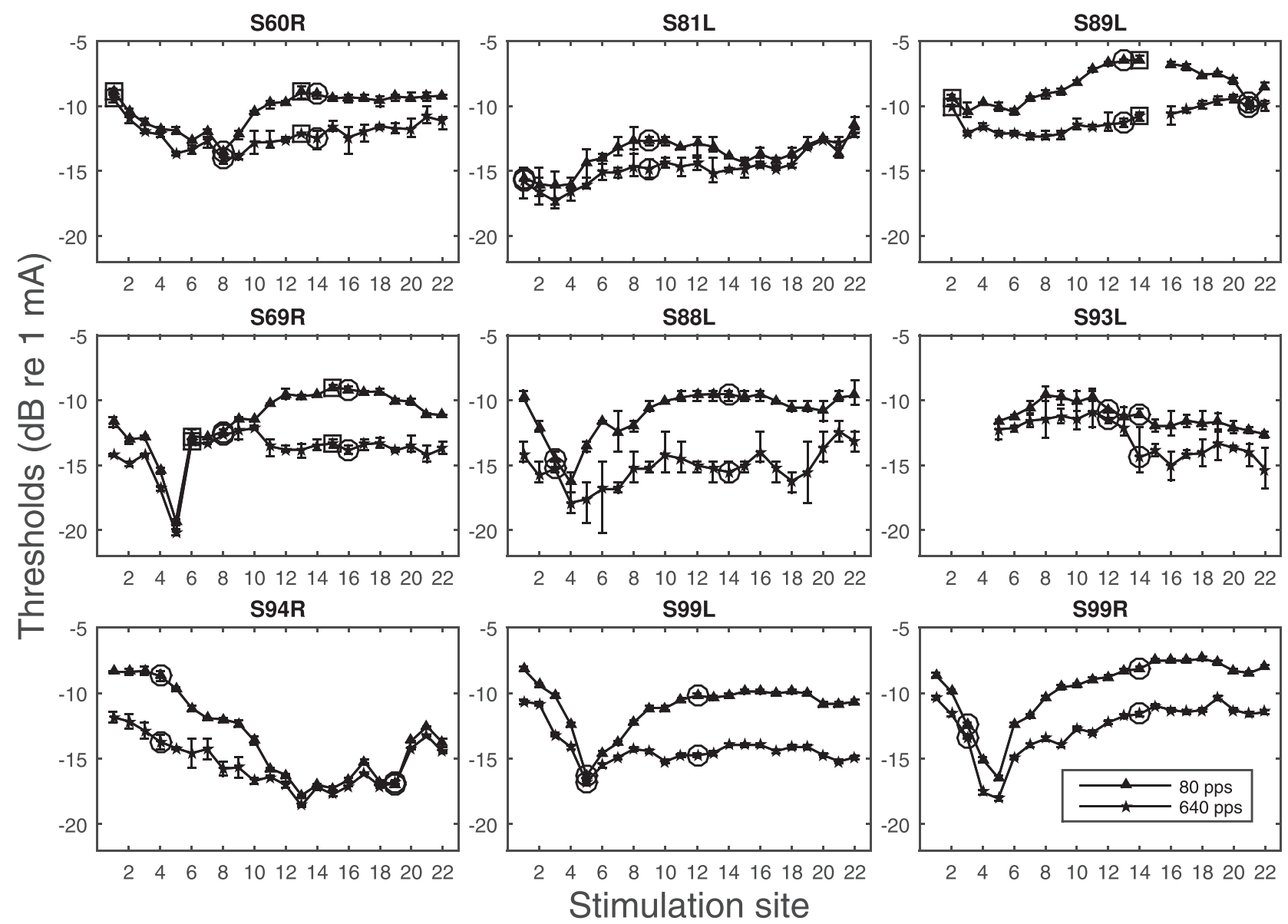

FIG. 1. Psychophysical detection thresholds measured for two stimulation rates across the electrode array. The error bars represent the range of the data (high and low thresholds). The stimulation sites chosen to be tested for spatial selectivity are indicated by circles. A second pair of electrodes was chosen for three subjects and is indicated by squares.

cases they were even non-monotonic. Forward masking, usually, but not always, gradually decayed as the distance of the masker from the probe increased. A general trend observed with the masking pattern was that the site with steeper MPI slopes showed broader neural excitation than the shallower-MPI site, except for case S93L, and one of the sets of sites in case of S60R (S60R set2). With data pooled across cases, a negative correlation between the MPI slopes and the forward-masking slopes was found (Fig. 5), with broader masking functions predicting steeper MPI slopes $[r(23)=-0.83, p<0.001]$. A univariate general linear model indicated that the slopes of the forward-masking function significantly accounted for the between-electrode variance in the MPI slopes $[F(1)=57.24, p<0.001]$, but did not account for the between-subject variance in the MPI slopes $[F(7)=1.335, p<0.301]$.

The masker levels were loudness balanced and therefore were different in magnitude across stimulation sites. On average, the absolute masker current levels varied across stimulation sites with a standard deviation of $0.84 \mathrm{~dB}(\sim 5$ CLU). If the absolute masker level at $0 \mathrm{~mm}$ was considerably higher at one probe location than another, peak masking at $0 \mathrm{~mm}$ could be greater at the probe site with the higher maker level. If the masking strength is comparable at the distant sites, the increased peak masking might artificially increase the sharpness of the masking functions. To determine the effect of variation in masker levels, the masker levels at $0 \mathrm{~mm}$ were compared between the sites with steep MPIs and those with shallow MPIs. A correlational analysis was also performed for the masker levels at $0 \mathrm{~mm}$ and the slopes of the masking functions. The absolute masking levels at $0 \mathrm{~mm}$ were not significantly different between the stimulation sites with steep MPI slopes and those with shallow MPI slopes $[t(11)=-0.97, p=0.35]$. Also, the masking levels at $0 \mathrm{~mm}$ were not correlated with the slopes of the masking functions $[r(23)=-0.34, p>0.05]$.

Figure 6 shows the relationship between the slopes of the masking functions and the electrode-modiolus distances of the corresponding stimulation sites estimated based on the average electrode to modiolus distance found in CT studies by Long and colleagues $(2014,2015)$. In the data from Long and colleagues, the high-resolution CT scans revealed a pattern from the base to apex in the CI24RE users, where the very basal electrodes (electrodes 1 and 2) were, on average, far from the modiolus, followed by a sharp decrease in distance around electrode 4, a gradual increase in distance that peaked around electrode 10, and then a gradual decrease in distance toward the apex. The stimulation sites tested here were assigned with estimated distance values based on electrode numbers of the stimulation sites. The correlation 


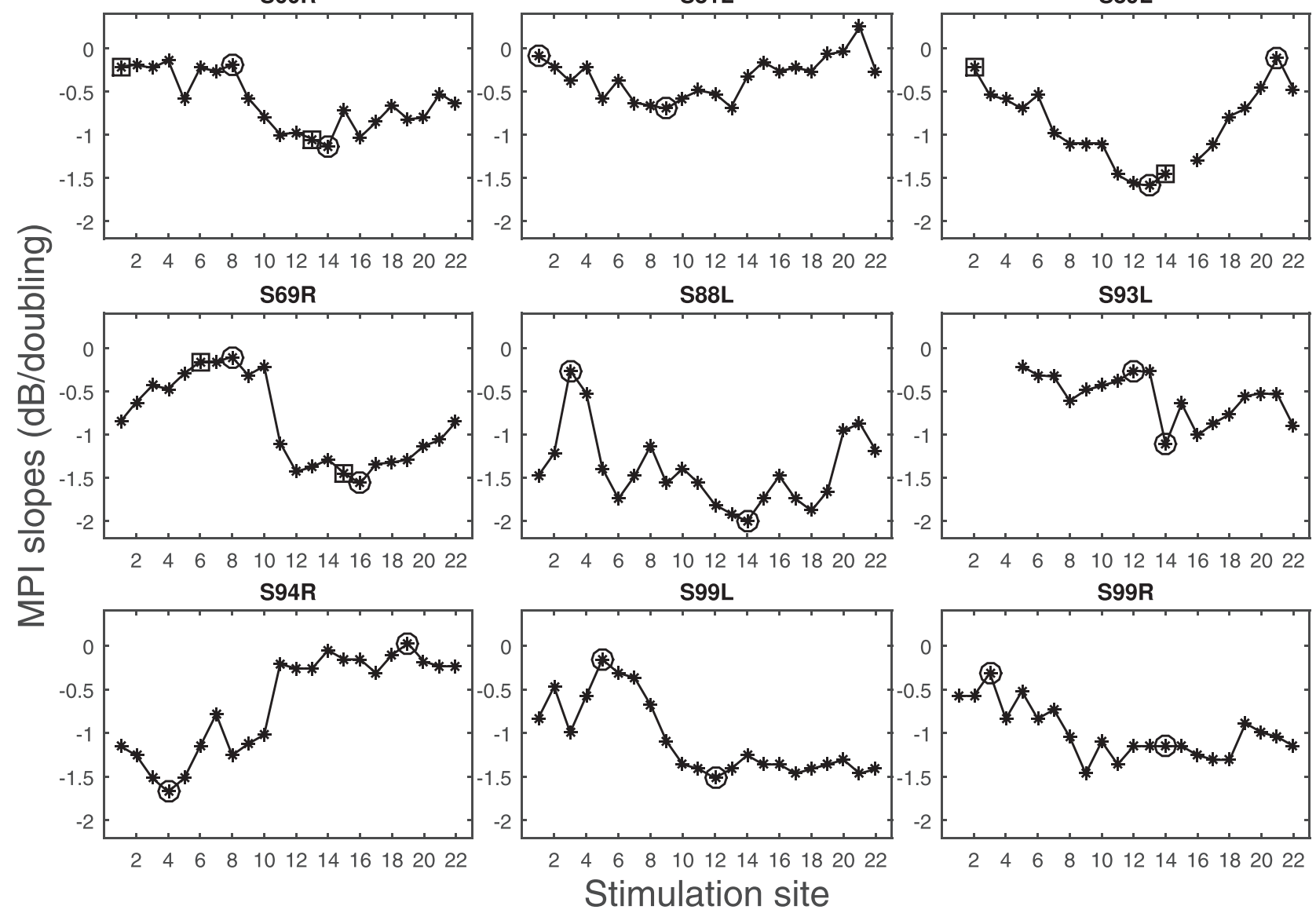

FIG. 2. MPI slopes as a function of stimulation site. Stimulation sites chosen to be further tested for forward-masking are indicated by circles. A second pair of electrodes was chosen for three subjects and is indicated by squares.

between the estimated distances from the electrodes to the modiolus and the slopes of the masking functions was marginally significant $[r(11)=0.39, p=0.06]$. Three stimulation sites (S69R, Site 8; S60R, Site 8; and S93L, Site 12) were estimated to have far distances but were measured to have moderately good spatial selectivity. It is possible that the three stimulation sites were at tonotopic locations that were more basal than what are typical for these electrode numbers; particularly for S93L, who had four basal electrodes deactivated, likely due to a shallow insertion. With S93L removed, the estimated distances agreed well with the psychophysically measured spatial selectivity as suggested by the significant linear fit $[r(11)=0.45, p=0.02]$. On average, the steep-MPI stimulation sites were estimated to have significantly greater electrode-modiolus distances than the shallow-MPI stimulation sites $[t(11)=2.25, p<0.05]$ (Fig. 6).

The relationship between the detection thresholds at the two tested rates and the slopes of the masking functions was also examined. Figure 7 shows that the detection thresholds at 80 pps were positively correlated with the slopes of the masking functions, with higher thresholds predicting broader spread of neural excitation $[r(23)=0.7, p<0.001]$, whereas there was no relationship between the detection thresholds at $640 \mathrm{pps}$ and the slopes of the masking functions $[r$ (23) $=0.19, p=0.36]$. Results of a univariate general linear model indicated that the 80-pps thresholds significantly accounted for both the between-electrode $[F(1)=112.31, p<0.001]$ and between-subject variance $[F(7)=7.87, p<0.001]$ in the slopes of the masking functions.

\section{DISCUSSION}

MPI is a psychophysical correlate of neural health in guinea pigs with CIs (Kang et al., 2010; Pfingst et al., 2011; Zhou et al., 2015). In these studies, steepness of the MPI functions correlated with the density of SGNs near the stimulation site, but other cochlear health variables, such as hair cell survival and the level of spontaneous activity in the fibers, co-varied with the SGNs and could also have contributed to the MPI slopes. Interestingly, in many human subjects where MPI varies across stimulation sites, there was no measurable residual hearing. Thus there were presumably no hair cells and no spontaneous activity in the auditory fibers in the electrically stimulated frequency regions (Zhou et al., 2012, 2015; Zhou and Pfingst, 2014 and this study) suggesting that hair cells are not required for MPI.

The present study aimed to further test the hypothesis that MPI slopes are dependent on the number of excitable neurons at the stimulation site. This was done by assessing MPI slopes as a function of spread of neural excitation for high pulse rate stimuli (900vpps) as determined by forwardmasking functions. In human subjects with CIs, the mediallateral position of the electrodes in the scala tympani varies as a function of length along the electrode array, whereas in 
S60R

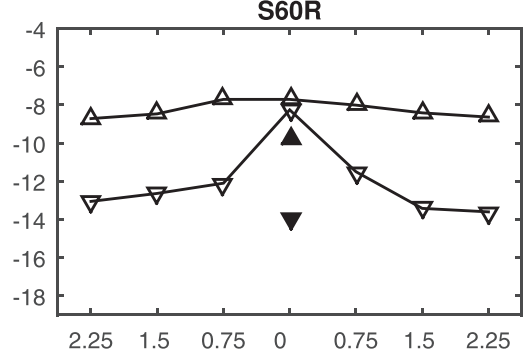

S89L

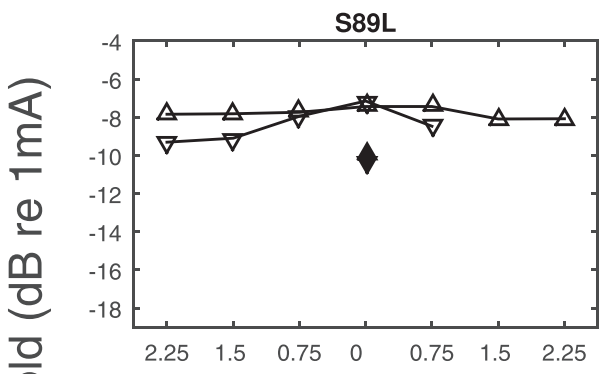

S69R set2

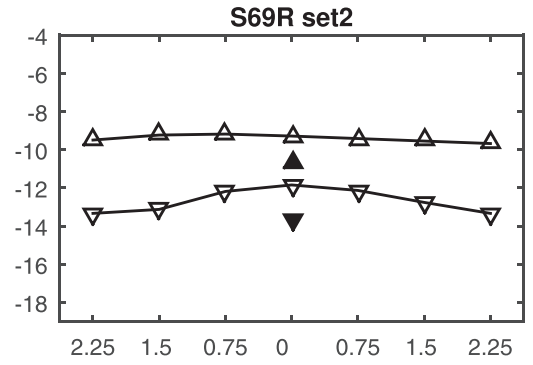

S94R

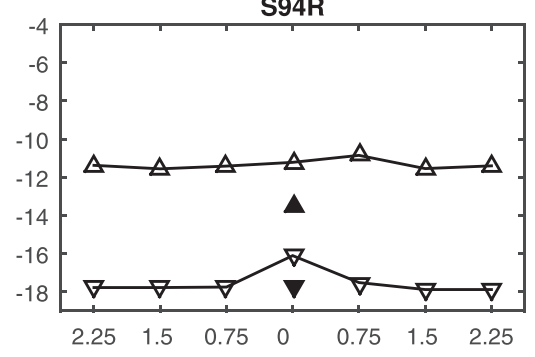

S60R set2

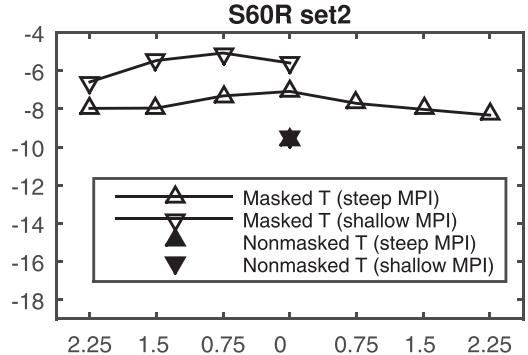

S89L set2

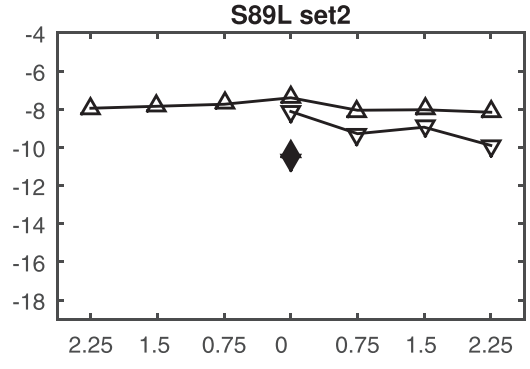

S88L

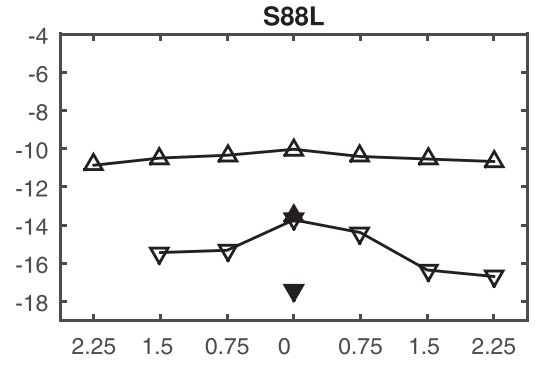

S99L

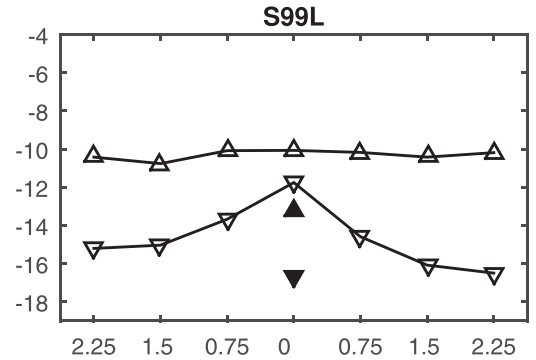

S81L

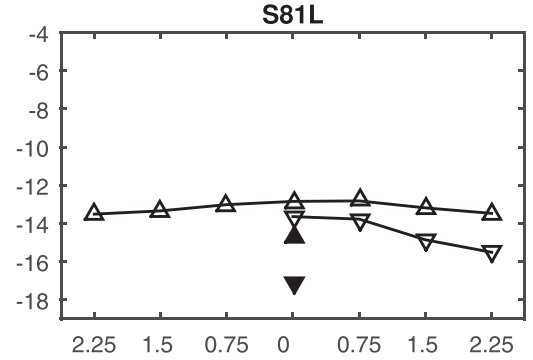

S69R

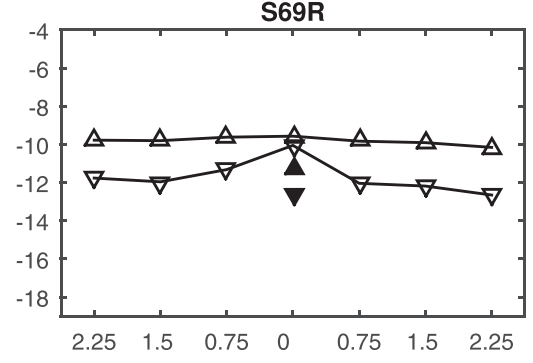

S93L

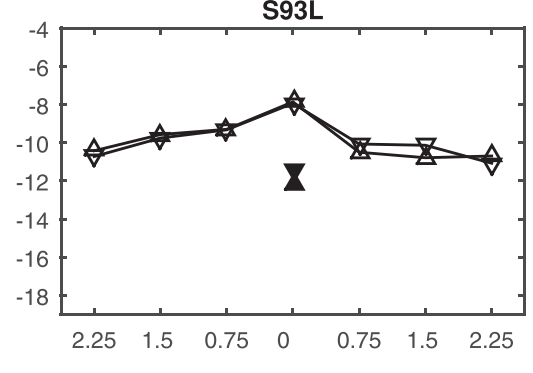

S99R

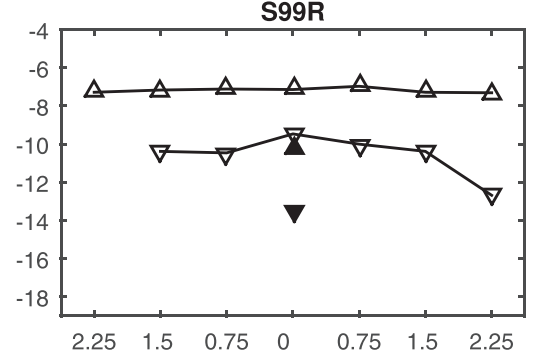

Masker to probe distance $(\mathrm{mm})$

FIG. 3. Non- and forward-masked detection thresholds at the probe stimulation sites. The filled triangles indicate non-masked probe thresholds and the open triangles indicate probe thresholds in the presence of a forward masker that was placed basal to, at, or apical to the probe location (from left to right). The uppointing triangles represent data measured at stimulation sites with the steep MPI, whereas the down-pointing triangles represent data measured at stimulation sites with the shallow MPI. Note that the number of masker sites was limited in cases where the probe site was close to either end of the electrode array. The conditions left to $0 \mathrm{~mm}$ in the figure were masker sites basal to the probe site.

the above cited animal studies the position of the electrode was fairly constant. Electrodes that are further from the modiolus can potentially activate a larger population of neurons thus introducing a new way to determine the relationship between the number of active neurons and MPI functions. The results of the current study indicate that more integration, i.e., steep MPI slopes was associated with broad neural excitation, and less integration, i.e., shallow MPI slopes, was associated with spatially restricted neural excitation.

\section{A. Slopes of the forward-masking functions}

The forward-masking functions with a fixed-position probe and maskers at various places surrounding the probe exhibited characteristics of nonlinearity in some cases and non-monotonicity in rare cases. That is, in some cases forward-masking did not gradually decay as a function of probe-masker distance (e.g., Fig. 3, S88L) or was sometimes higher in the presence of a more-remote masker than a lessremote one (e.g., Fig. 3, S89L set2). This could possibly reflect uneven neural survival or irregularities in the current field among the masker sites. Note that for measuring each masking function, the level of all maskers was adjusted to equate to a designated loudness (as in McKay, 2012). For a masker site that has sparse neural survival, the masker probably needs to produce excitation away from its physical location to achieve the required loudness, thus the masker would produce more masking at the probe site compared to the situation if the masker produces excitation close to its physical location (Cosentino et al., 2015). Using loudness balanced 

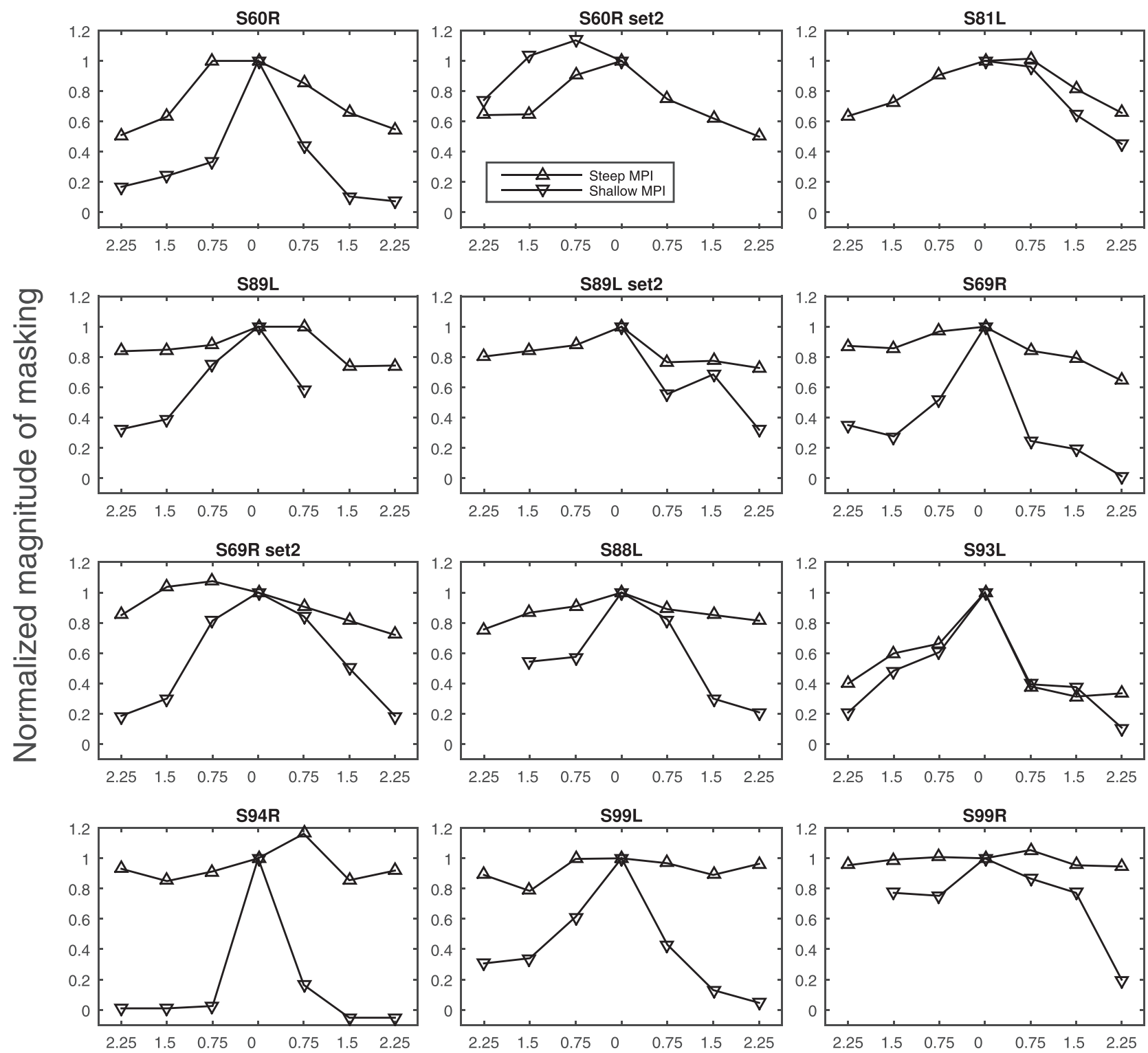

Masker to probe distance $(\mathrm{mm})$

FIG. 4. Normalized forward-masking functions. The magnitude of forward-masking (masked minus non-masked thresholds) was normalized by dividing each data point by the magnitude of masking at $0 \mathrm{~mm}$. The up-pointing triangles indicate data measured at the stimulation sites with the steep MPI, whereas the down-pointing triangles indicate data measured at the stimulation sites with the shallow MPI.

maskers, the condition of the masker sites could add noise to the slope of the masking functions as a measure for spread of neural excitation, but this confounding factor probably affected the current data set minimally since the variation in the absolute masker levels was small (standard deviation $=0.8 \mathrm{~dB}$ ). The absolute masker levels at the $0 \mathrm{~mm}$ location did not contribute to the sharpness of the masking functions either. That is, higher masker levels at the $0 \mathrm{~mm}$ position were not associated with sharper masking functions. A shift of masking peak was sometimes observed with the relatively shallow masking functions (S60R, set2 S69R, set2), with an off-site masker being more effective than the masker placed on the same electrode as the probe. This suggested that the probe signal produced more excitation on the adjacent masker site than the probe site thus becoming more prone to the masking produced by the off-site masker. Another factor that could affect the sharpness of the masking function was the possible confusion between the masker and probe when they were presented at the same electrode. We know that temporal resolution varies considerably across sites and ears (Garadat and Pfingst, 2011) and $10 \mathrm{~ms}$ might not always be long enough to be perceived as a gap. When the masker and the probe are of similar loudness, likely at the beginning of the adaptive procedure, they could be heard as a single sound (Neff, 1986). This confusion might elevate the masked probe threshold, which would artificially sharpen the masking function. A close examination of the raw tracking data that showed the levels at which the reversals occurred suggested that this potential confusion did not affect the current data set.

\section{B. Relationship between MPI and the masking functions}

Despite the possible noise in the masking functions, a strong negative correlation was observed between the slopes 


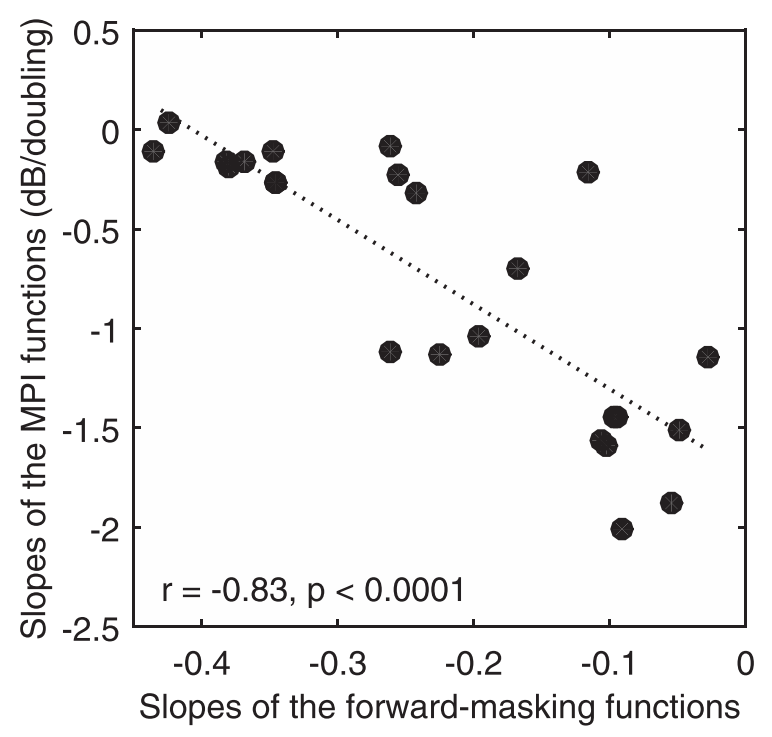

FIG. 5. Scatter plot of slopes of the MPI functions against the slopes of the forward-masking functions. The slopes of the forward-masking function were calculated as the normalized amount of forward-masking (function divided by peak masking at $0 \mathrm{~mm}$ ) per $\mathrm{mm}$ separation between the probe and masker. Each data point represents one stimulation site $(n=24)$. The dotted line shows the linear fit to the data. The correlation coefficient and the $p$ values are shown in the lower left corner of the panel.

of the masking functions measured at $900 \mathrm{pps}$ and the MPI slopes (Fig. 4). That is, a wider spread of neural excitation at a high rate predicted steeper MPI slopes. A spread of neural excitation can occur under two circumstances: distant stimulation or substantial nerve loss. With substantial nerve loss, higher current levels are required to achieve the desired loudness and this results in a greater spatial spread of activating currents. Distant stimulation produces a wide radiant of current spread and this may similarly result in a spatial spread of neural activation. In this study, the electrodeneuron distances for the stimulation sites of interest were estimated based on their electrode numbers using the averaged electrode-modiolus distances reported by Long and colleagues (2014, 2015). Long and colleagues (2014, 2015) have shown that the electrode-modiolus distance could vary widely, from 0.1 to $1.8 \mathrm{~mm}$ in humans, and vary systematically as a function of tonotopic location in many CI24RE (CA) users. The estimated distances in our subjects agreed well with the slopes of the forward-masking functions,

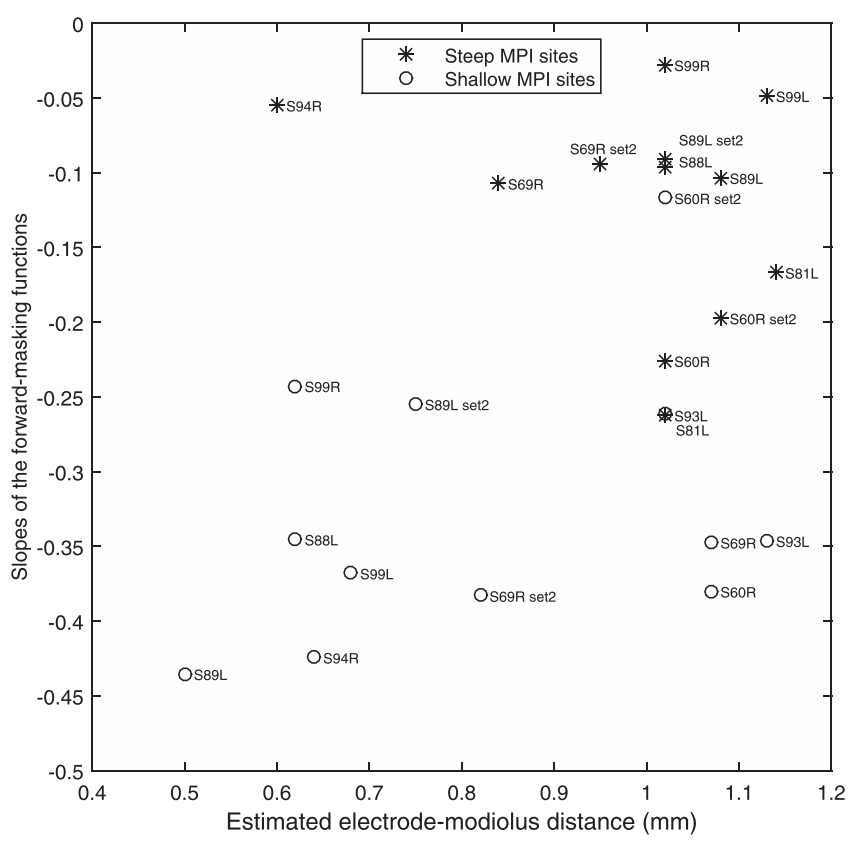

FIG. 6. Scatter plot of the slopes of the forward-masking functions against the estimated electrode-modiolus distances. Estimates of electrode-modiolus distances are based on data from Long and colleagues (2014, 2015). The stimulation sites with relatively shallow MPI slopes (open circles) or relatively steep MPI slopes (asterisks) are indicated. Each data point is labeled with the subject number.

suggesting that the broader masking patterns seen in this sample were generally associated with further electrode positions with respect to the modiolus. The stimulation sites with broader excitation patterns and estimated with larger electrode-modiolus distance were those that produced steeper MPI slopes. Below we offer two possible explanations that might account for this relationship.

First, we propose that the correlation between MPI and spread of excitation is related to the fact that the field of available neurons is larger at a site with a greater electrodemodiolus distance than one that receives narrower stimulation. The slope of the MPI function should correspond to the increase of neural activity in the temporal integration window as the stimulus, at a fixed current level, is increased in rate. A greater increase in neural activity with rate should predict a larger threshold decrease. As the stimulation rate is increased, neural excitability of single fibers will be limited
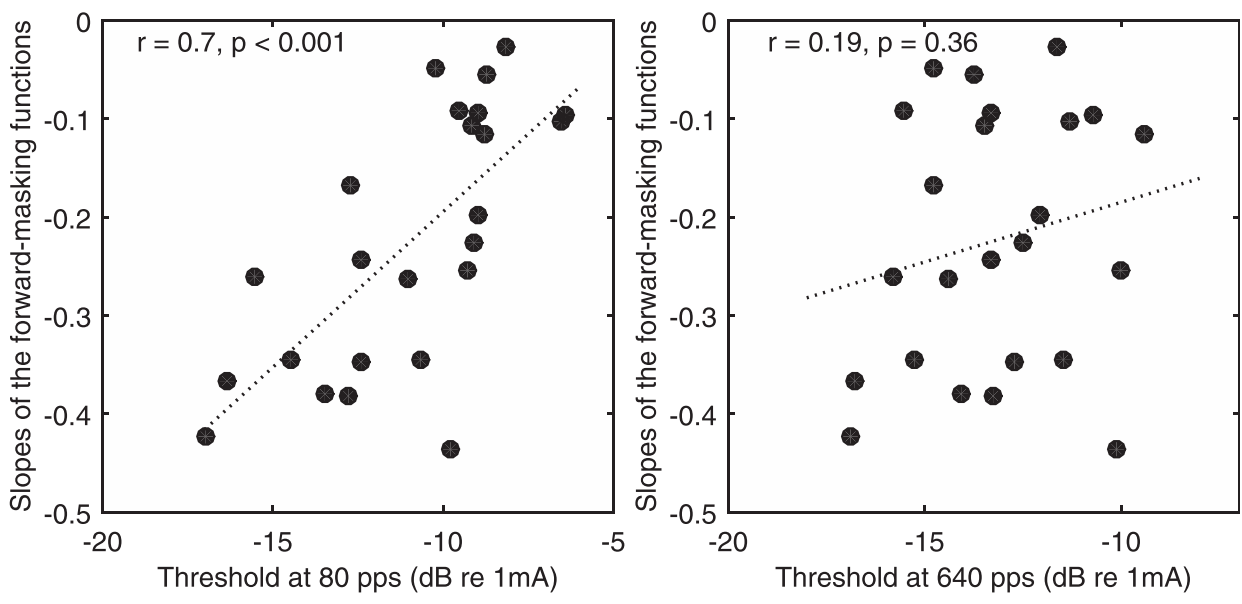

FIG. 7. Scatter plots of the slopes of the forward-masking functions against the 80-pps (left) and 640-pps (right) detection thresholds. Each data point represents one stimulation site $(n=24)$. The dotted line shows the linear fit to the data. The correlation coefficients and the $p$ values are shown in the upper left corner of each panel. 
by refractoriness and various forms of adaptation, making it unlikely for a single fiber to respond each time a pulse occurs (e.g., Smith and Brachman, 1982; Eggermont, 1985; Parkins, 1989; Javel and Shepherd, 2000; Javel and Viemeister, 2000; Miller et al., 2011; McKay et al., 2013). It is possible that the activated neurons at some stimulation sites are more temporally responsive than others, due to differences in the fibers' recovery time constants or neural health (Zhou et al., 1995), but it would be difficult to rationalize why neural health would be systematically better at sites with greater electrode-neuron distance. If, however, the fact that the electrode is distant from the neurons makes it possible to activate a greater number of neurons as the stimulation rate increases, then neural activity would increase in a greater ratio with rate compared to a site where the field of available neurons is small. This explanation is based on an assumption that the number of neurons being activated increases as the stimulation rate increases. It is possible that the neurons in the center of the current field respond to lowrate stimulation but as rate increases to a point where the excitability of those neurons is reduced by refractoriness and adaptation, neurons at the more periphery location of the current field would respond to stimulation. The current data could not be used to prove such an assumption because the spread of neural excitation was only assessed at a high rate (i.e., 900 pps), thus it would only reveal a neural excitation pattern similar to one detecting the 640 pps stimulus. If this assumption is true, then at threshold, the total number of neural spikes required to reach threshold could be achieved by recruiting a large number of neurons and driving them at a low current level producing an average low firing probability (low threshold at high rate), or stimulating a small number of neurons at a high current level producing a high firing probability (high threshold at low rate). This proposed scenario is more likely to occur at stimulation sites with large electrode-modiolus distance, where the current field is wide and excitation is more likely to spread than one receiving narrow stimulation.

Indeed, shallow MPI slopes were observed at stimulation sites estimated to have small electrode-to-modiolus distances. The sharp valleys in threshold versus place functions at those sites observed in some subjects, i.e., S69R, S87L, S99L, S99R (Fig. 1) suggest that stimulation produced by the close-distance electrodes with monopolar stimulation might be similar to that produced by focused stimulation. As the stimulation rate increases, the small population of neurons at the more focused stimulation sites must increase their spiking rate to achieve a lowered threshold level, assuming not enough additional neurons could be recruited. Poststimulus time histograms measured from single auditory fibers suggest that at a low physical current level, activity of single fibers essentially remains the same despite an increase of stimulation rate from 200 to $1000 \mathrm{pps}$ (Heffer et al., 2010). At a relatively high physical current level, an increase in stimulation rate increases the fibers' discharge rate at the stimulus onset but response quickly adapts over the course of the stimulus (Heffer et al., 2010). The single-fiber physiology data suggest that zero MPI slopes are also possible, if the rate increase is not accompanied by spread of excitation. There was only one case, i.e., S93L, electrode 14, where a steep MPI slope did not require a broad neural excitation. The neural density local to the stimulation site was perhaps exceptionally high, although this was not commonly observed at least in the current data set. There were also two basal stimulation sites (S60R/E1; S81L/E1, Fig. 2) that showed broad masking patterns but produced shallow MPI slopes. It is possible that these electrodes, located at regions where neural degeneration usually first occurs with hearing loss, stimulated a sizable area with poor nerve survival. Therefore the broad masking patterns observed at these sites indicate that the active neurons were spatially spread out, but does not necessarily suggest the number of the activated neurons was large. In summary, the above proposed explanation assumes that a steep threshold decrease with increasing stimulation rate would only occur if the field of available neurons is large enough to produce increased neural activity with the increase of stimulation rate.

An alternative explanation is that, assuming invariant spread of excitation with rate, the steepness of the MPI slope is related to the slope of the composite neural I/O function at the stimulation site. A steep I/O function would predict a shallow MPI slope, because, to maintain threshold, a smaller current reduction would be needed to compensate for an increase in stimulation rate if neural excitation grows steeply with current level. One likely example is S60R/E1. In addition to a small MPI slope in that example, the absolute thresholds were also high. It is possible that the high threshold current activated the more densely packed neurons in the internal auditory meatus or auditory fibers with steep I/O functions, such that neural excitation increased steeply with current change. This would predict a shallow MPI slope, but would not necessarily suggest that a small number of neurons were able to be recruited.

\section{Relationship between detection thresholds and spread of neural excitation}

At the stimulation sites estimated to contrast in electrode-modiolus distances, the absolute detection thresholds were also often indicative of spatial selectivity at the respective sites, with higher thresholds predicting shallowsloped forward-masking patterns. However, this relationship only held true for thresholds at 80 pps (Fig. 5). For S60R set2 and S93L, the two stimulation sites had comparable low-rate thresholds (Fig. 1), and comparable masking function slopes (Fig. 3), but very different high-rate thresholds. In some extreme cases, e.g., S89L set2, S69R, S69R set2, and S88L, the 640-pps threshold was lower for the stimulation site with a considerably broader spread of excitation than that with a narrower spread of excitation. These results were consistent with the inverse relationship between the rate of threshold decrease with stimulation rate (MPI) and spread of excitation observed in the present study. That is, because threshold decreased much more steeply with rate by broad stimulation, low thresholds for high-rate stimulus might sometimes indicate poor spatial selectivity of neural excitation.

\section{Implication for speech recognition}

The results of the present study showed a trading relationship between spread of excitation and MPI in the human 
subjects. With focused stimulation, while tuning may be sharp, neural representation of the spectral patterns produced by the high-rate stimuli might not be accurate. This might explain why there has not been consistent evidence indicating that focused stimulation is better than monopolar stimulation for speech recognition, using carrier frequencies close to those tested here, i.e., 640 pps (e.g., Pfingst et al., 2001; Srinivasan et al., 2013). It should be noted however that pulse rate discrimination at supra-threshold levels such as those used for speech recognition is not necessarily governed by the same mechanisms as threshold change as a function of pulse rate.

Results from Zhou and Pfingst (2014) showed that the ear differences in MPI slopes predicted ear differences in speech recognition, with steep MPI predicting better speech recognition performance. The current results suggest that steep MPI slopes were associated with broad neural excitation at a stimulation rate close to that used in the clinical maps. It should be noted that when taking the ear differences in the MPI and speech recognition measures, the effects of subjectspecific variables such as cognition were removed and only the ear-specific variables remained. If we assume that the electrode-neuron distance versus place pattern is an earspecific variable, then the ear with a steeper average MPI slope would be the ear with poor spatial selectivity of neural excitation. If spatial selectivity of neural excitation is important for speech recognition (Won et al., 2007), then steeper MPI slopes might predict worse speech recognition in that ear, which was not the case. It is possible that the poor spatial selectivity of neuron excitation in the ear with steep MPI slopes traded for more activated neurons per channel, which might have helped coding the more temporally dynamic speech cues such as those for place of articulation of consonants. This interpretation is consistent with the results showing a strong correlation between MPI and perception of place of articulation. Assuming that the electrode-neuron distance versus place pattern is a subject variable, that is, if the insertion patterns of the electrode array are similar between the two ears in the same subject, then the ear differences in the MPI slopes should only reflect the ear differences in neural survival with steep slopes predicting better neural survival. This is consistent with the findings that the ear differences in the MPI slopes were able to predict those in speech recognition in noise in general and consonant recognition, but it cannot explain why they failed to predict vowel recognition. The relationship between MPI and speech recognition warrants further investigation.

\section{CONCLUSION}

In conclusion, MPI slopes are dependent on having a sufficient number of auditory fibers representing the highrate stimulus. In the current data set, steep MPI slopes were observed frequently at stimulation sites with a broad spread of excitation but rarely at sites with a narrow spread. The data suggest that in humans without residual acoustic hearing and a partially depleted neural population, accurate neural representation of high rate stimuli relies on stimulating relatively broad regions in the cochlea.

\section{ACKNOWLEDGMENT}

We thank our dedicated CI subjects for their participation. This work was supported by NIH-NIDCD Grant Nos. R01 DC010786 and P30 DC005188, and an Emerging-Research Grant from the Hearing Health Foundation.

Azadpour, M., AlJasser, A., and McKay, C. M. (2013). "Place specificity measured in forward and interleaved masking in cochlear implants," J. Acoust. Soc. Am. 134, EL314-EL320.

Boulet, J., White, M., and Bruce, I. C. (2016). "Temporal considerations for stimulating spiral ganglion neurons with cochlear implants," J. Assoc. Res. Otolaryngol. 17, 1-17.

Carlyon, R. P., Deeks, J. M., and McKay, C. M. (2015). "Effect of pulse rate and polarity on the sensitivity of auditory brainstem and cochlear implant users to electrical stimulation,” J. Assoc. Res. Otolaryngol. 16, 653-668.

Cosentino, S., Deeks, J., and Carlyon, R. P. (2015). "Spatial selectivity: How to measure and (maybe) improve it," in Conference on Implantable Auditory Prostheses, Abstract W51.

Eggermont, J. J. (1985). "Peripheral auditory adaptation and fatigue: A model oriented review," Hear. Res. 18, 57-71.

Garadat, S. N., and Pfingst, B. E. (2011). "Relationship between gap detection thresholds and loudness in cochlear-implant users," Hear. Res. 275, 130-138.

Heffer, L. F., Sly, D. J., Fallon, J. B., White, M. W., Shepherd, R. K., and O'Leary, S. J. (2010). "Examining the auditory nerve fiber response to high rate cochlear implant stimulation: Chronic sensorineural hearing loss and facilitation," J. Neurophysiol. 104, 3124-3135.

Hu, N., Abbas, P., Miller, C., Robinson, B., Nourski, K., Jeng, F., Abkes, B., and Nichols, J. (2003). "Auditory response to intracochlear electric stimuli following furosemide treatment," Hear. Res. 185, 77-89.

Hughes, M. L., Baudhuin, J. L., and Goehring, J. L. (2014). "The relation between auditory-nerve temporal responses and perceptual rate integration in cochlear implants," Hear. Res. 316, 44-56.

Hughes, M. L., Castioni, E. E., Goehring, J. L., and Baudhuin, J. L. (2012). "Temporal response properties of the auditory nerve: Data from human cochlear-implant recipients," Hear. Res. 285, 46-57.

Javel, E., and Shepherd, R. K. (2000). "Electrical stimulation of the auditory nerve. III. Response initiation sites and temporal fine structure," Hear. Res. 140, 45-76.

Javel, E., and Viemeister, N. F. (2000). "Stochastic properties of cat auditory nerve responses to electric and acoustic stimuli and application to intensity discrimination,” J. Acoust. Soc. Am. 107, 908-921.

Kang, S. Y., Colesa, D. J., Swiderski, D. L., Su, G. L., Raphael, Y., and Pfingst, B. E. (2010). "Effects of hearing preservation on psychophysical responses to cochlear implant stimulation," J. Assoc. Res. Otolaryngol. 11, 245-265.

Long, C. J., Holden, T. A., McClelland, G. H., Parkinson, W. S., Shelton, C., Kelsall, D. C., and Smith, Z. M. (2014). "Examining the electro-neural interface of cochlear implant users using psychophysics, CT scans, and speech understanding," J. Assoc. Res. Otolaryngol. 15, 293-304.

Long, C. J., Melman, R. O., Parkinson, W. S., Holden, T. A., Potts, W. B., and Smith, Z. M. (2015). "Investigating the electro-neural interface: The effects of electrode position and age on neural responses," in Conference on Implantable Auditory Prostheses, Abstract S4.

McKay, C. M. (2012). "Forward masking as a method of measuring place specificity of neural excitation in cochlear implants: A review of methods and interpretation," J. Acoust. Soc. Am. 131, 2209-2224.

McKay, C. M., Chandan, K., Akhoun, I., Sicilliano, C., and Kluk, K. (2013). "Can ECAP measures be used for totally objective programming of cochlear implants?,” J. Assoc. Res. Otolaryngol. 14, 879-890.

McKay, C. M., Henshall, K. R., Farrell, R. J., and McDermott, H. J. (2003). "A practical method of predicting the loudness of complex electrical stimuli,” J. Acoust. Soc. Am. 113, 2054-2063.

Middlebrooks, J. C. (2004). "Effects of cochlear-implant pulse rate and inter-channel timing on channel interactions and thresholds," J. Acoust. Soc. Am. 116, 452-468.

Miller, C. A., Woo, J., Abbas, P. J., Hu, N., and Robinson, B. K. (2011). "Neural masking by sub-threshold electric stimuli: Animal and computer model results," J. Assoc. Res. Otolaryngol. 12, 219-232.

Neff, D. L. (1986). "Confusion effects with sinusoidal and narrow-band noise forward maskers," J. Acoust. Soc. Am. 79, 1519-1529. 
Parkins, C. W. (1989). "Temporal response patterns of auditory nerve fibers to electrical stimulation in deafened squirrel monkeys," Hear Res. 41, 137-168.

Pfingst, B. E., Colesa, D. J., Hembrador, S., Kang, S. Y., Middlebrooks, J. C., Raphael, Y., and Su, G. L. (2011). "Detection of pulse trains in the electrically stimulated cochlea: Effects of cochlear health," J. Acoust. Soc. Am. 130, 3954-3968.

Pfingst, B. E., Franck, K. H., Xu, L., Bauer, E. M., and Zwolan, T. A. (2001). "Effects of electrode configuration and place of stimulation on speech perception with cochlear prostheses," J. Assoc. Res. Otolaryngol. 2, 87-103.

Ramekers, D., Versnel, H., Strahl, S. B., Klis, S. F., and Grolman, W. (2015). "Recovery characteristics of the electrically stimulated auditory nerve in deafened guinea pigs: Relation to neuronal status," Hear. Res. 321, 12-24.

Smith, R. L., and Brachman, M. L. (1982). "Adaptation in auditory-nerve fibers: A revised model," Biol. Cybern. 44, 107-120.

Srinivasan, A. G., Padilla, M., Shannon, R. V., and Landsberger, D. M. (2013). "Improving speech perception in noise with current focusing in cochlear implant users," Hear Res. 299, 29-36.
Viemeister, N. F., and Wakefield, G. H. (1991). "Temporal integration and multiple looks," J. Acoust. Soc. Am. 90, 858-865.

Wilson, B. S., Finley, C. C., Lawson, D. T., and Zerbi, M. (1997). "Temporal representations with cochlear implants," Am. J. Otol. 18, S30-S34.

Won, J. H., Drennan, W. R., and Rubinstein, J. T. (2007). "Spectral-ripple resolution correlates with speech reception in noise in cochlear implant users,” J. Assoc. Res. Otolaryngol. 8, 384-392.

Zhou, R., Abbas, P. J., and Assouline, J. G. (1995). "Electrically evoked auditory brainstem response in peripherally myelin-deficient mice," Hear. Res. 88, 98-106.

Zhou, N., Kraft, C. T., Colesa, D. J., and Pfingst, B. E. (2015). "Integration of pulse trains in humans and guinea pigs with cochlear implants," Assoc. Res. Otolaryngol. 16, 523-534.

Zhou, N., and Pfingst, B. E. (2014). "Relationship between multipulse integration and speech recognition with cochlear implants," J. Acoust. Soc. Am. 136, 1257-1268.

Zhou, N., Xu, L., and Pfingst, B. E. (2012). "Characteristics of detection thresholds and maximum comfortable loudness levels as a function of pulse rate in human cochlear implant users," Hear. Res. 284, 25-32. 